\title{
Reliability Analysis of Tape Winding Hydraulic System Based on Continuous-Time T-S Dynamic Fault Tree
}

\author{
Honghua Sun, ${ }^{1,2}$ Lipeng $X u \mathbb{D}^{1},{ }^{1}$ Guangjun Jiang, ${ }^{1,2}$ and Hongxia Chen $\mathbb{D}^{1,2}$ \\ ${ }^{1}$ Inner Mongolia University of Technology, Hohhot 010051, Inner Mongolia, China \\ ${ }^{2}$ Inner Mongolia Key Laboratory of Advanced Manufacturing Technology, Hohhot 010051, Inner Mongolia, China \\ Correspondence should be addressed to Hongxia Chen; 734429229@qq.com
}

Received 8 January 2022; Revised 30 January 2022; Accepted 3 February 2022; Published 26 February 2022

Academic Editor: Gianpaolo Di Bona

Copyright ( $\odot 2022$ Honghua Sun et al. This is an open access article distributed under the Creative Commons Attribution License, which permits unrestricted use, distribution, and reproduction in any medium, provided the original work is properly cited.

It is necessary to make the tape winding system have good reliability. Because the power system usually is a pneumatic or hydraulic system, the static reliability analysis lacks the ability to describe the dynamic state transfer, component fault correlation, and propagation effect of the system; it is difficult to accurately reflect the actual behavior of the complex system. Most of the existing research results lack the situation that the importance of bottom events changes with time; only a single number cannot express the impact of bottom events on top events at different times. Therefore, this study uses the dynamic reliability analysis method to quantitatively analyze the reliability of the tape winding power hydraulic system. Firstly, the dynamic reliability model of hydraulic system is established by using the continuous-time T-S dynamic fault tree to solve the fault rate of the system. Secondly, the results are compared with the traditional Markov chain analysis method of dynamic fault tree. The feasibility and advantages of the continuous-time T-S dynamic fault tree analysis method are verified. Finally, the probability importance and key importance of the system unit are calculated, and the law of importance changing with time is expressed. According to the importance of different time, it can help the regular maintenance and fault diagnosis of equipment.

\section{Introduction}

Winding products have important applications in the fields of aerospace, national defense, and petrochemical industry and are the key to reducing weight and improving performance [1]. During the manufacturing process, a certain amount of pressure is required to make the materials tightly intertwined in a predetermined shape to ensure good tightness. As a key technology in tape winding, the pressure system is applied to the tape to enhance the bonding between the layers of the tape and remove the bubbles, improve the bonding strength between the tape layer and the density of products, and prevent layer fold and slip effect, to improve the strength of the products. Among them, too little pressure will lead to the tape and the laminated plate cannot reach the close bonding state, which affects the mutual penetration of the polymer chain; excessive pressure will extrude the collective in the molten state from the laminate, resulting in the phenomenon of poor glue [2], which cannot ensure the good adhesion of the tape belt because the working environment of the tape winding needs a certain temperature to ensure that the impregnation will not solidify in advance to maintain the viscous state. It also provides stable pressure to maintain the tightness of the winding process. Nowadays, the more commonly used power systems are pneumatic systems and hydraulic systems.

This paper takes the hydraulic system as the research object for reliability analysis. On this basis, the importance analysis method is used to provide help for system fault diagnosis and reliability allocation. Because there are many influencing factors of faults in the hydraulic system, its structure is complex, there are many components, and it is difficult to ensure its good reliability when the hydraulic system is used as the power supply system. The modern hydraulic system becomes lighter and more complex. Although the traditional fault tree analysis method is one of the modern, more extensive, and perfect reliability analysis methods and can carry out some static event simple logic 
reliability analysis of the hydraulic system, it lacks the ability to describe the dynamic state transfer of the system and the correlation of component faults. Compared with the traditional fault tree analysis, the dynamic fault tree reliability analysis can better express the sequence and correlation between events, make the results have certain practical significance, and reduce the deviation and misleading to the system design and maintenance. Based on the dynamic fault tree, the proposed T-S dynamic fault tree analysis method can describe the static and dynamic failure logic relationship of any form and multistate [3] when solving the reliability problem of the hydraulic system and is more concise than the general method of dynamic fault tree analysis, which can directly carry out quantitative calculation.

Based on the T-S dynamic fault tree, the continuoustime T-S dynamic fault tree analysis method has a stronger ability to describe the failure behavior compared with the Dugan dynamic fault tree. The continuous-time T-S dynamic fault tree is an analysis and calculation method based on the fault probability density function and fault probability distribution function of the parent event, which describes the rule execution degree and the occurrence probability of the parent event, as well as the impulse function integral, by using the integral characteristics of the impulse point of the impulse function [4].

In modern engineering, various systems interact with each other, the complexity is increasing, and the requirements for reliability are also improving. The traditional static fault tree analysis cannot meet the description and analysis of system dynamic changes and state transition. Since 1990-1992, Dugan, an international reliability scholar, has extended the traditional fault tree; since a group of dynamic gates are defined to describe the dynamic failure behavior of the system, the leap from static to dynamic reliability analysis is realized [5], which opens a new chapter for reliability analysis.

It is difficult to analyze the reliability of complex systems by using the dynamic fault tree. After years of research by reliability scholars, there are many solutions, such as the Markov chain method for fault tree analysis of repairable and unrepairable systems, and the conversion process is simplified [6-8]. However, with the increase of system fault factors and the complexity of logical relations, the difficulty of Markov calculation also increases exponentially, so the method is not applicable; then, the Bayesian network algorithm is proposed to alleviate the data explosion problem and the calculation difficulty of Markov chain $[9,10]$; to reflect the variation law of system reliability with time, the dynamic fault tree is transformed into discrete-time Bayesian network, and the efficiency of solving the dynamic fault tree is improved by reasoning algorithm $[11,12]$. Then, a series of dynamic reliability analyses under continuous time are proposed; in engineering practice, the state of the system is full of uncertainty, including random uncertainty and cognitive uncertainty. To make the reliability analysis results more realistic, fuzzy theory, interval theory, and other analysis methods appear, the probability distribution of dynamic logic gate under the fuzzy tree is established, and the calculation method of system fuzzy failure rate is given
$[13,14]$; Monte Carlo simulation method is used for reliability calculation to describe the dynamic changes of the system $[15,16]$.

Dugan dynamic fault tree logic gate cannot describe the relationship among all dynamic events of hydraulic components because of the many and complex components in the hydraulic system in practical engineering, and it also has some limitations in describing the combined failure behaviors of components. The T-S dynamic fault tree analysis method proposed by Takagi and Sugeno has a stronger failure description ability for reliability analysis of the hydraulic system and can express both dynamic and static states breaking through the limitations of previous dynamic fault trees and making the reliability of hydraulic system closer to reality.

T-S dynamic fault tree reliability analysis has many application examples, such as the establishment of the T-S system dynamic fuzzy fault tree, which provides a method to find the weak link of the hydraulic system of the loader [17]. The reliability analysis model of the hydraulic system based on the T-S fuzzy fault tree model is established, and the fusion Monte Carlo numerical simulation method is used to realize the simulation of system reliability. Finally, the effectiveness of the method is verified by a typical hydraulic circuit [18]. The T-S fuzzy fault tree and Bayesian network method are transformed to make a reasonable evaluation of the reliability of tunnel collapse, which provides a method for the safety construction decision of tunnel [19]. In order to overcome the deficiency of dealing with the uncertainty of fuzzy information and fault logic relationship in the traditional importance measurement, the T-S fuzzy importance analysis method is proposed and applied to the importance analysis of the hydraulic system, which proves the feasibility of the algorithm [20].

In [17-19], only the reliability analysis of the system was carried out, and the influence of a single bottom event fault on the top event was not judged. In [20], importance was applied to analyze the bottom events, but it cannot reflect the change of importance over time. In this study, the continuous-time T-S dynamic fault tree is used to analyze the reliability of the hydraulic system of the tape winding machine. Then, the importance of each bottom event is analyzed by the importance of the continuous-time T-S dynamic fault tree. It is pointed out that the weak links of the system provide help for system reliability optimization, distribution, and fault diagnosis.

\section{Continuous-Time T-S Dynamic Fault Tree Analysis Method}

The research of the T-S fault tree makes the fault tree analysis method stronger in describing system faults, but it cannot reflect the changes of today's complex system reliability with time. Then, a discrete-time T-S dynamic fault tree analysis method is developed. Due to the discrete-time T-S dynamic fault tree itself, there are calculation errors in the analysis, which cannot reflect the probability change trend of system reliability. With the increase of time interval, the calculation error decreases and the amount of calculation increases. 
When it tends to infinity, the results are not different from the results of continuous-time T-S dynamic fault tree analysis. Therefore, the continuous-time T-S dynamic fault tree is proposed to solve the problem that system reliability changes with time and fault logic is difficult to describe, which makes up for the deficiency of the discrete-time T-S dynamic fault tree. Continuous-time T-S dynamic fault tree is an analysis and calculation method of upper-level event, fault probability density function, and fault probability distribution function based on the integral characteristics of the impulse points of impulse function and describing the degree of rule execution, the possibility of upper-level events, and the integral of impulse function.

\subsection{Analysis Steps}

(1) Refer to the literature, the working principle of the system, and the experience and the lessons of experts to determine the top event, intermediate event, and basic events and establish the dynamic fault tree reliability model.

(2) The logic gates in the dynamic fault tree model are transformed into a continuous-time T-S dynamic fault tree model by regular description according to the correlation of events and time logic order.

(3) Consider the uncertainty description methods of events, such as random probability and ambiguity. Then, establish the possibility of rule execution.

(4) The continuous-time T-S dynamic fault tree analysis and calculation method is used to obtain the fault probability of the top event as shown in Figure 1.

Transformation from dynamic fault tree to T-S dynamic fault tree in Figure 2.

In Figure 2, $F$ represents the top event, $X_{1}$ and $X_{2}$ represent the bottom events causing $F, S$ represents the static logic, and $T$ represents the dynamic logic. As shown in the figure, the T-S dynamic fault tree has the advantage of describing the logical relationship between dynamic and static states, which only needs to be described by different rules. The number " $r$ " of rules is related to the number $n$ of subordinate events $X_{i}$, which can be expressed by the following formula:

$$
r=n !+1+\sum_{i=1}^{n-2} C_{n}^{n-i}((i+1) !) .
$$

The rule description is constructed based on the logical relationship of events and the timing of the failure. Among them, each rule represents a temporal relationship, using " 1 , $2, \ldots, n$ " to represent the occurrence sequence of basic events. The smaller the number is, the more priority the event occurs, $t_{1}<t_{2}<\ldots<t_{\mathrm{n}}$, for example, $X_{1}=1, X_{2}=2$, which means that event $X_{1}$ occurs before $X_{2}$. If the numbers are the same, it is considered that events occur at the same time. The failure time of the subordinate event $X_{i}$ and the superior event $F$ are represented by $t_{i}$ and $t$, respectively, and constrained by the impulse function, as shown in the following formula:

$$
\delta\left(t-t_{i}\right)= \begin{cases}\infty, & t=t_{i}, \\ 0, & t \neq t_{i},\end{cases}
$$

where $t$ is time and $t_{i}$ is the moment of the impulse point. If $\delta\left(t-t_{i}\right)=\infty$, that is, $\mathrm{t}=t_{i}$, it means event $F$ fault is after $X_{i}$ fault. The following rules for T-S dynamic gates will be described with AND-gate, priority AND-gate, and OR-gate as examples; the system is assumed to be a two-state system. If 0 and 1 are used to represent normal and fault states, the possibility of top events occurring at different times is represented by $0,1.0$ is impossible, and 1 is possible.

(1) AND-gate: According to the logical nature of the occurrence of bottom events, the occurrence of top events is described in the following table. When bottom events occur at the same time, that is $t_{1}=t_{2}$, the occurrence of top events occurs; if the occurrence of events is sequential, the top event occurs after the occurrence of both bottom events. The description method is shown in Table 1.

(2) Priority AND-gate: The priority AND-gate has a time sequence; when the bottom events occur in a certain order, the top event will occur. The rules are described as follows: assuming that $X_{1}$ occurs first at $t_{1}$ time and then $X_{2}$ occurs at $t_{2}$ time, the top event will occur when $t_{1}<t_{2}$; otherwise, the top event will not occur. When two bottom events occur at the same time, the top event occurs. The description method is shown in Table 2.

(3) OR-gate: When one of the bottom events occurs, the top event will occur. Its rules are described as shown in Table 3: when the bottom event occurs at the same time, the top event occurs; if the bottom event occurs in a certain time series, when the bottom event $X_{i}$ occurs first, the top event occurs at time $t$.

Through the above examples, it can be seen that, compared with traditional fault tree analysis and dynamic fault tree analysis, this method can succinctly express the timing and logical relationship of complex system faults by using the size relationship between numbers and simply describe all situations of system faults, to make the reliability fit the actual working situation.

2.2. Continuous-Time T-S Dynamic Fault Tree Algorithm. After the transformation of the rule description, the T-S dynamic fault tree is analyzed and solved. Use $f_{l}\left(X_{i}^{t_{i}}\right)$ to represent the event at a lower level under the rule of $L$ failure probability density function, the instantaneous failure rate in the moment $t_{i}$ is expressed as $\lambda\left(X_{i}^{t_{i}}\right)$, and the said possibility of rule execution is expressed as $P_{L}^{*}$. Then, the probability of rule $L$ execution is

$$
P_{L}^{*}=\prod_{i=1}^{n} f_{i}\left(X_{i}^{t_{i}}\right) .
$$

If the failure rate changes between the subordinate events in the continuous-time T-S dynamic gate due to the 


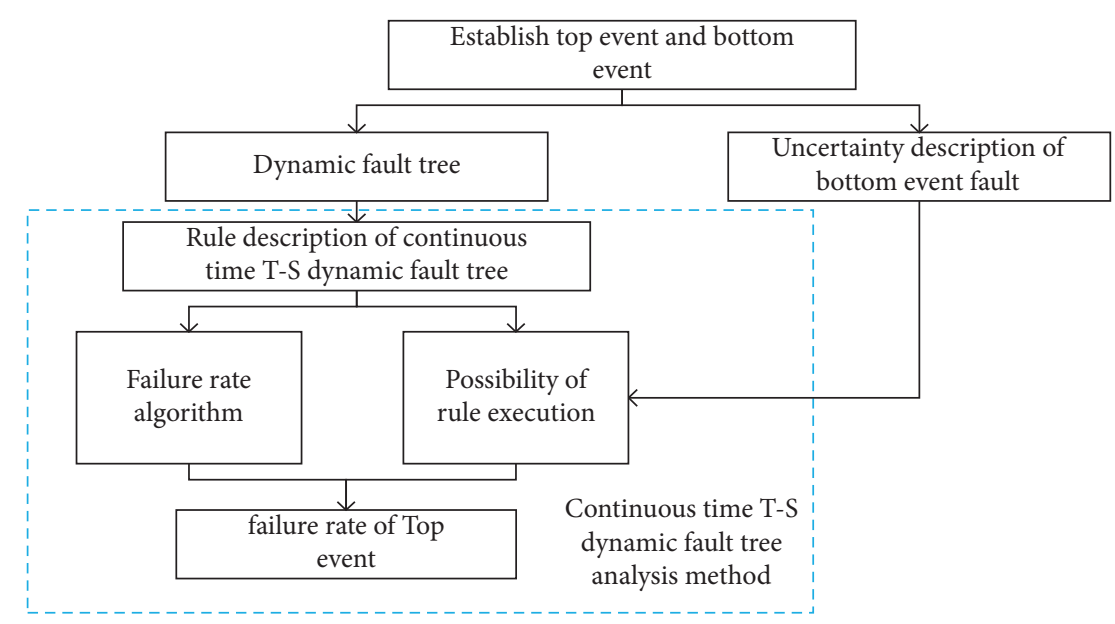

FIGURE 1: Continuous-time T-S dynamic fault tree analysis method.

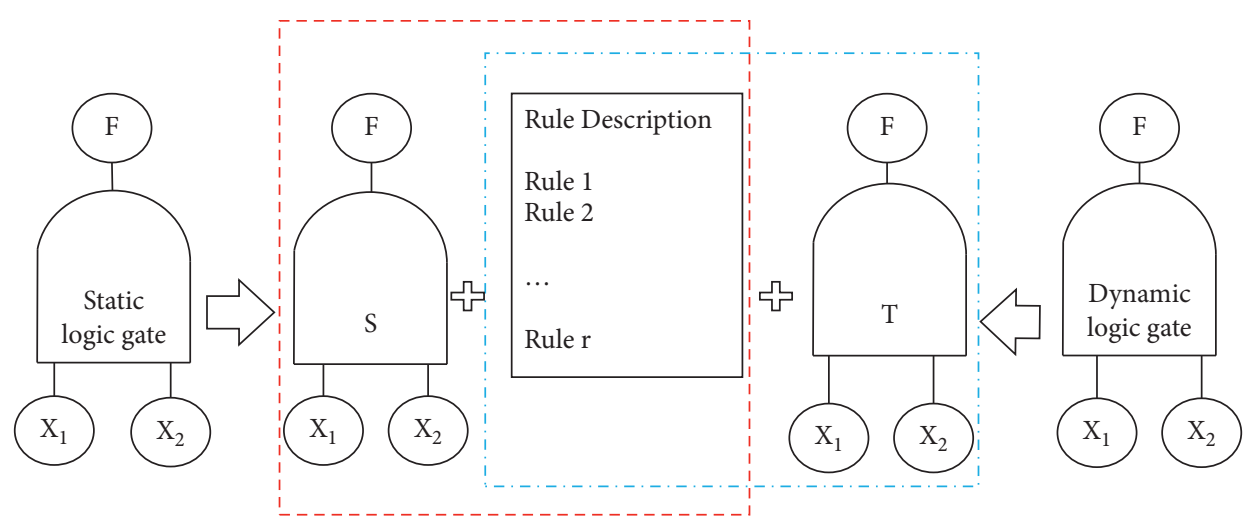

Figure 2: Conversion of static and dynamic logic gates to T-S dynamic gates.

TABLE 1: Rule description of AND-gate.

\begin{tabular}{lcccc}
\hline Rule & $X_{1}$ & $X_{2}$ & $Y\left(t-t_{1}\right)$ & $Y\left(t-t_{2}\right)$ \\
\hline 1 & 1 & 1 & 1 & 1 \\
2 & 1 & 2 & 0 & 1 \\
3 & 2 & 1 & 1 & 0 \\
\hline
\end{tabular}

TABLE 2: Rule description of priority AND-gate.

\begin{tabular}{lcccc}
\hline Rule & $X_{1}$ & $X_{2}$ & $Y\left(t-t_{1}\right)$ & $Y\left(t-t_{2}\right)$ \\
\hline 1 & 1 & 1 & 1 & 1 \\
2 & 1 & 2 & 0 & 1 \\
3 & 2 & 1 & 0 & 0 \\
\hline
\end{tabular}

TABLE 3: Rule description of OR-gate.

\begin{tabular}{lcccc}
\hline Rule & $X_{1}$ & $X_{2}$ & $Y\left(t-t_{1}\right)$ & $Y\left(t-t_{2}\right)$ \\
\hline 1 & 1 & 1 & 1 & 1 \\
2 & 1 & 2 & 1 & 0 \\
3 & 2 & 1 & 0 & 1 \\
\hline
\end{tabular}

fault timing, then the probability density function of the subordinate events under rule $L$ is

$$
f_{i}\left(X_{i}^{t_{i}}\right)=\lambda_{i}\left(X_{i}^{t_{i}}\right) \exp \left(\int_{0}^{t_{i}}-\lambda_{i}\left(X_{i}^{\tau}\right) \mathrm{d} \tau\right) .
$$

If the event failure rate is at a lower level with a fixed value $\lambda_{i}$, there is no fault correlation between events $X_{i}$ at a lower level; namely, event $X_{i}$ at a lower level of any one failure will not affect other events at a lower level failure probability of change, the probability density function of event $X_{i}$ can be expressed as: $f_{i}\left(X_{i}^{t_{i}}\right)=f\left(X_{i}^{t_{i}}\right)$. Therefore, formula (4) can be expressed as

$$
f\left(X_{i}^{t_{i}}\right)=\lambda_{i} \exp \left(\int_{0}^{t_{i}}-\lambda_{i} \mathrm{~d} \tau\right)
$$

Superior events under the rules of $L$, the $t_{i}$ moment when the possibility of failure can be expressed as $P_{L}\left(y^{t_{i}}\right)$, and the top event fault probability density function of " $y$ " are as follows:

$f_{y}(t)=\sum_{L=1}^{r} \sim \iint \ldots \int \sum_{i=1}^{n} \delta\left(t-t_{i}\right) P_{L}^{*} P_{L}\left(y^{t_{i}}\right) \mathrm{d} t_{1} \mathrm{~d} t_{2} \ldots \mathrm{d} t_{n}$.

The integral interval for $\Omega_{l}(l=1,2, \ldots, r)$. In the formula, " $r$ " represents the number of rules, " $n$ " represents the 
number of subordinate events causing the occurrence of superior events, and the integral interval is, respectively, expressed as

$$
\begin{aligned}
& \Omega_{1}\left\{\left(t_{1}, t_{2}, \ldots, t_{n}\right) \mid 0<t_{1}=t_{2}=\ldots=t_{n}\right\}, \\
& \Omega_{2}\left\{\left(t_{1}, t_{2}, \ldots, t_{n}\right) \mid 0<t_{1}=t_{2}=\ldots=t_{n-1}<t_{n}\right\}, \\
& \Omega_{r}\left\{\left(t_{1}, t_{2}, \ldots, t_{n}\right) \mid 0<t_{n}<t_{n-1}<\ldots<t_{1}\right\} .
\end{aligned}
$$

The probability distribution function of the top event is

$$
F_{y}(t)=\int_{0}^{t} f_{y}(t) \mathrm{d} t
$$

According to the formula and rule description of the continuous-time T-S dynamic fault tree analysis algorithm above, the occurrence probability of the intermediate events can be obtained, and then the occurrence probability of the top event can be gained. Finally, the reliability analysis results of the system can be obtained. In the calculation process, the bottom event occurs at the same time, for example, $t_{1}=t_{2}$. This failure mode has a very low probability or is even impossible to occur in the actual project, so it can be omitted in the calculation of system reliability.

\section{The Application of Continuous-Time T-S Dynamic Fault Tree}

Taking the hydraulic power system of a winding trolley as an example, the continuous-time T-S dynamic fault tree is used to analyze the dynamic reliability, and the results are compared with those of the dynamic fault tree Markov chain analysis. The hydraulic principle of the winding trolley is shown in Figure 3.

The hydraulic system of tape winding machine mainly includes oil cylinder, hydraulic pump, oil pipe, and relevant valve body, which can be divided into two functional parts: first, control the movement of the winding trolley to complete the movement of the winding path; second, control the movement of the hot pressing roller. The hot pressing roller carries the tape belt extrusion die to give the required pressure during winding. The pressure can be adjusted through the overflow valve. The hydraulic system consists of three hydraulic cylinders, a double-acting piston, a single rod oil cylinder for moving the winding trolley, and two jacks for jacking up the hot pressing roll. Only the fault and normal working states of the hydraulic system are considered.

According to the working principle of the hydraulic system, firstly, the hydraulic oil reaches the quantitative hydraulic pump through the oil filter through the oil pipe. Secondly, the hydraulic oil flows through the overflow valve according to the required oil quantity and adjusts the pressure. Finally, the hydraulic oil passes through the reversing valve at a certain pressure to control the movement of the trolley and hot pressing roller. A dynamic fault tree is established by taking the fault of the control loop of the hot press roll as the top event as shown in Figure 4, where $y$ indicates that the control circuit of the hot pressing roller is faulty, $\mathrm{Y}_{1}, \mathrm{Y}_{2}$, and $\mathrm{Y}_{3}$, respectively, indicate that the oil of the reversing valve is abnormal, the hydraulic cylinder is damaged, and the oil is abnormal, $G_{1}, G_{2}$, and $G_{3}$ represent OR-gate, $G_{4}$ represents priority AND-gate, and the names of each bottom event element and its failure rate $\left(\lambda_{i}\right)$ are shown in Table 4.

Assume that the hydraulic system is a two-state system and the working time of the system is $t=10000 \mathrm{~h}$; the above dynamic fault tree is transformed into a T-S dynamic fault tree. $S_{1}, S_{2}$, and $S_{3}$ represent OR-gate and $T_{4}$ represents priority AND-gate, as shown in Figure 5.

Referring to the instance in Section 2, the T-S dynamic gate rules are calculated as follows.

Take $T_{4}$ priority AND-gate as an example; because $T_{4}$ consists of two bottom events $X_{5}$ (the tubing) and $X_{6}$ (oil filter) and one top event $\mathrm{Y}_{3}$ (the oil is abnormal), the rule description of $T_{4}$ is calculated according to formula (1):

$$
r=2 !+1+\sum_{i=1}^{0} C_{2}^{2-i}((i+1) !)=3
$$

Therefore, the number of rules of $S_{3}$ and $S_{1}$ can be calculated. The number of rules of $\mathrm{S}_{2}$ can also be calculated according to formula (1):

$$
r=3 !+1+\sum_{i=1}^{1} C_{3}^{3-i}((i+1) !)=13 .
$$

So, the rules of $T_{4}, S_{3}, S_{2}$, and $S_{1}$ can be described in Tables 5-8.

Use the continuous-time T-S dynamic fault tree analysis algorithm described in Section 2 to analyze and calculate the failure rates of the intermediate events and the top event $Y$.

Taking $T_{4}$ as an example, the failure rate of each event can be obtained in Table 4. According to formula (5), the probability density function of bottom events $X_{5}$ and $X_{6}$ can be expressed as

$$
\begin{aligned}
& f\left(X_{5}^{t_{5}}\right)=\lambda_{5} \exp \left(\int_{0}^{t_{5}}-\lambda_{5} \mathrm{~d} \tau\right)=\lambda_{5} \exp \left(-\lambda_{5} t_{5}\right) \\
& f\left(X_{6}^{t_{6}}\right)=\lambda_{6} \exp \left(\int_{0}^{t_{6}}-\lambda_{6} \mathrm{~d} \tau\right)=\lambda_{6} \exp \left(-\lambda_{6} t_{6}\right) .
\end{aligned}
$$

So, the possibility of rule execution $P_{L}^{*}$ can be expressed by formula (3) as

$$
P_{L}^{*}=f\left(X_{5}^{t_{5}}\right) f\left(X_{6}^{t_{6}}\right)
$$

In the $T_{4}$ priority AND-gate, the probability density function of top event $\mathrm{Y}_{3}$ can be obtained by simultaneous formulas (6) and (8) and the rules in Table 5. 


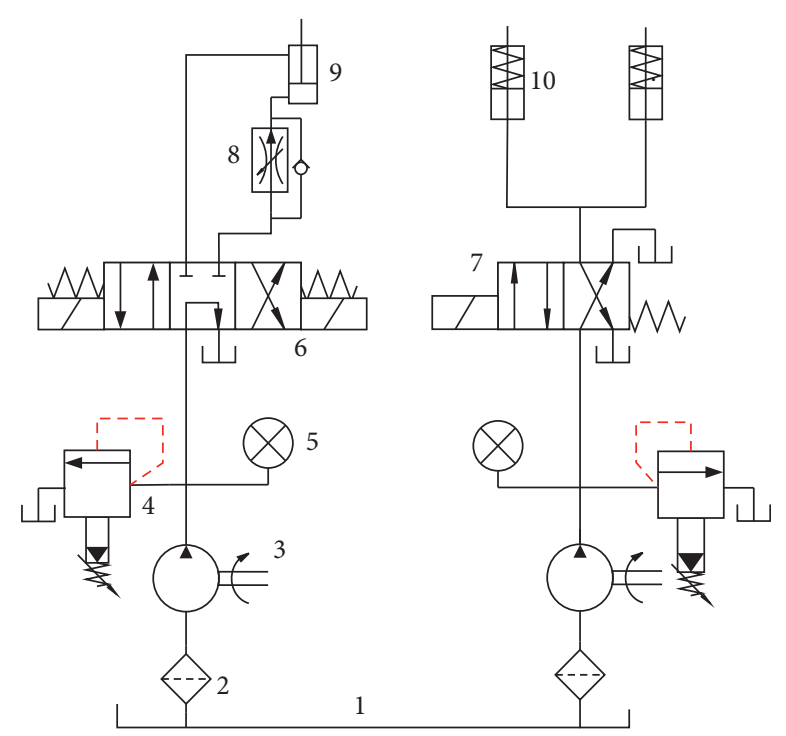

Figure 3: Hydraulic schematic diagram of winding machine. 1, oil tank; 2, oil filter; 3, quantitative hydraulic pump; 4, relief valve; 5, pressure gauge; 6 , three-position four-way solenoid valve; 7 , two-position four-way solenoid valve; 8 , one-way speed regulating valve; 9 , winding car lifting hydraulic cylinder; 10, thin Jack.

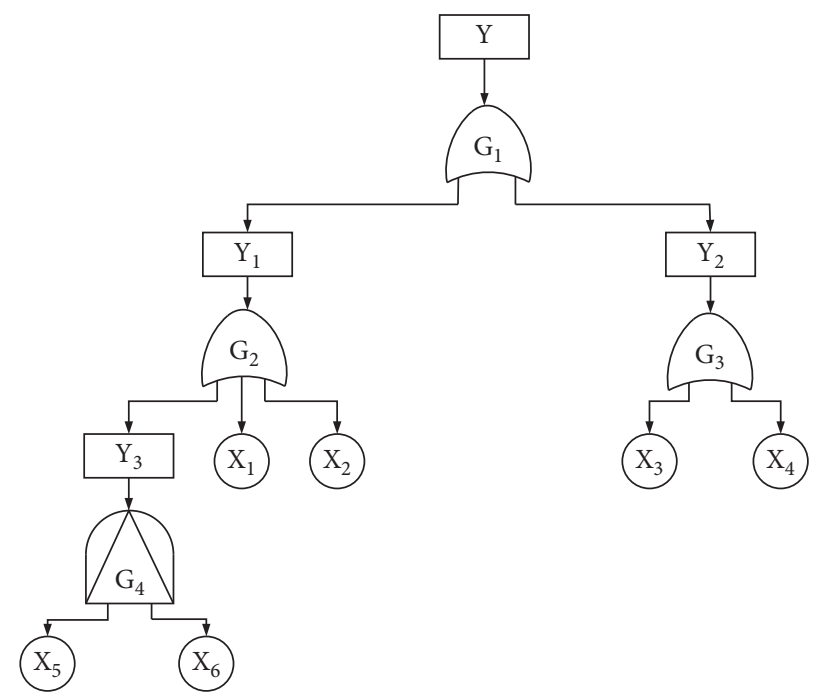

FIgURE 4: Dynamic failure tree of hot pressing roller control system.

TABLE 4: Components corresponding to bottom events and failure rate.

\begin{tabular}{lcc}
\hline The bottom event $\left(X_{i}\right)$ & Name of the event & Failure rate $\left(\lambda_{i}\right)\left(\times 10^{-6} / h\right)$ \\
\hline$X_{1}$ & Quantitative hydraulic pump & 25.3 \\
$X_{2}$ & The overflow valve & 13.8 \\
$X_{3}$ & Directional control valve & 18.4 \\
$X_{4}$ & Jack & 12.6 \\
$X_{5}$ & The tubing & 15.7 \\
$X_{6}$ & Oil filter & 19.5 \\
\hline
\end{tabular}




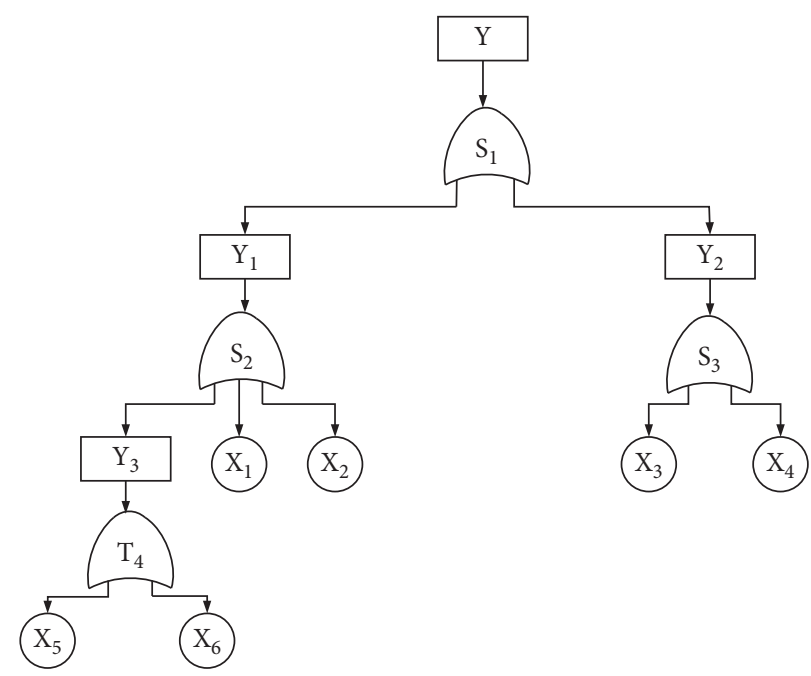

Figure 5: T-S dynamic fault tree.

TABle 5: Rule description of $T_{4}$.

\begin{tabular}{lcccr}
\hline Rule & $X_{5}$ & $X_{6}$ & $Y_{3}\left(t_{y_{3}}-t_{\mathbf{x}_{5}}\right)$ & $Y_{3}\left(t_{y_{3}}-t_{\mathbf{x}_{6}}\right)$ \\
\hline 1 & 1 & 1 & 1 & 1 \\
2 & 1 & 2 & 0 & 1 \\
3 & 2 & 1 & 0 & 0 \\
\hline
\end{tabular}

TABLe 6: Rule description of $S_{3}$.

\begin{tabular}{lcccc}
\hline Rule & $X_{3}$ & $X_{4}$ & $Y_{2}\left(t_{y_{2}}-t_{\mathbf{x}_{3}}\right)$ & $Y_{2}\left(t_{y_{2}}-t_{\mathbf{x}_{4}}\right)$ \\
\hline 1 & 1 & 1 & 1 & 1 \\
2 & 1 & 2 & 1 & 0 \\
3 & 2 & 1 & 0 & 1 \\
\hline
\end{tabular}

Table 7: Rule description of $S_{2}$.

\begin{tabular}{|c|c|c|c|c|c|c|}
\hline Rule & $Y_{3}$ & $X_{1}$ & $X_{2}$ & $Y_{1}\left(t_{y_{1}}-t_{y_{3}}\right)$ & $Y_{1}\left(t_{y_{1}}-t_{x_{1}}\right)$ & $Y_{1}\left(t_{y_{1}}-t_{x_{2}}\right)$ \\
\hline 1 & 1 & 1 & 1 & 1 & 1 & 1 \\
\hline 2 & 1 & 1 & 2 & 1 & 1 & 0 \\
\hline 3 & 1 & 2 & 1 & 1 & 0 & 1 \\
\hline 4 & 2 & 1 & 1 & 0 & 1 & 1 \\
\hline 5 & 1 & 2 & 2 & 1 & 0 & 0 \\
\hline 6 & 2 & 1 & 2 & 0 & 1 & 0 \\
\hline 7 & 2 & 2 & 1 & 0 & 0 & 1 \\
\hline 8 & 1 & 2 & 3 & 1 & 0 & 0 \\
\hline 9 & 1 & 3 & 2 & 1 & 0 & 0 \\
\hline 10 & 2 & 1 & 3 & 0 & 1 & 0 \\
\hline 11 & 2 & 3 & 1 & 0 & 0 & 1 \\
\hline 12 & 3 & 2 & 1 & 0 & 0 & 1 \\
\hline 13 & 3 & 1 & 2 & 0 & 1 & 0 \\
\hline
\end{tabular}

TABle 8: Rule description of $S_{1}$.

\begin{tabular}{lcccc}
\hline Rule & $Y_{1}$ & $Y_{2}$ & $Y\left(t_{y}-t_{\mathbf{y}_{1}}\right)$ & $Y\left(t_{y}-t_{\mathbf{y}_{2}}\right)$ \\
\hline 1 & 1 & 1 & 1 & 1 \\
2 & 1 & 2 & 1 & 0 \\
3 & 2 & 1 & 0 & 1 \\
\hline
\end{tabular}




$$
\begin{aligned}
f_{y_{3}}(t) & =\int_{0}^{t} \int_{0}^{+\infty} \delta\left(t-t_{6}\right) \lambda_{5} \exp \left(-\lambda_{5} t_{5}\right) \times \lambda_{6} \exp \left(-\lambda_{6} t_{6}\right) \times 1 \mathrm{~d}_{t_{5}} \mathrm{~d}_{t_{6}} \\
& =\int_{0}^{t} \lambda_{5} \exp \left(-\lambda_{5} t_{6}\right) \times \lambda_{6} \exp \left(-\lambda_{6} t_{6}\right) \mathrm{d}_{t_{6}}=-\frac{\lambda_{5} \times \lambda_{6}}{\lambda_{5}+\lambda_{6}} \exp \left[\left(-\lambda_{5}-\lambda_{6}\right) t\right]+\frac{\lambda_{5} \times \lambda_{6}}{\lambda_{5}+\lambda_{6}} .
\end{aligned}
$$

Therefore, the probability distribution function of $Y_{3}$ can be got according to formula (7):

$$
\begin{aligned}
F_{Y_{3}}(t)= & \int_{0}^{t} f_{y_{3}}(t) \mathrm{d}_{t}=\frac{\lambda_{5} \times \lambda_{6}}{\left(\lambda_{5}+\lambda_{6}\right)^{2}} \exp \left[\left(-\lambda_{5}-\lambda_{6}\right) t\right] \\
& +\frac{\lambda_{5} \times \lambda_{6}}{\lambda_{5}+\lambda_{6}} t-\frac{\lambda_{5} \times \lambda_{6}}{\left(\lambda_{5}+\lambda_{6}\right)^{2}} .
\end{aligned}
$$

When different values of $t$ are given, the top event failure rate at the corresponding time can be obtained. For example, when $t=10000 \mathrm{~h}$, the failure rate of $Y_{3}$ is 0.013659 .

In summary, the calculated failure rate of the hydraulic system $Y$ is 0.034375 . The curve of failure rate changing with time is shown in Figure 6 . The $x$-axis represents the time $(\mathrm{t} / \mathrm{h})$, and the $y$-axis represents the failure rate.

Next, the conventional algorithm of the dynamic fault tree is applied to transform the dynamic fault tree into a Markov chain, and the fault rate of the fault tree is solved by the state transition equation:

$$
\left\{\begin{array}{l}
X_{1}+X_{2}+X_{3}+X_{4}, \\
X_{5} \longrightarrow X_{1}+X_{2}+X_{3}+X_{4}+X_{6} \\
X_{6} \longrightarrow X_{5} \longrightarrow X_{1}+X_{2}+X_{3}+X_{4} \\
X_{6} \longrightarrow X_{1}+X_{2}+X_{3}+X_{4} .
\end{array}\right.
$$

Take the third way as an example. When the system works normally, it enters another working state due to $X_{6}$ fault and then $X_{5}$ fault. However, since $X_{5}$ and $X_{6}$ form a logic priority and gate for the top event fault, according to $X_{6}$ $\longrightarrow X_{5}$, the system will not cause system failure. Therefore, the system enters the next state and continues to work. When any bottom event of $X_{1}, X_{2}, X 3$, and $X_{4}$ fails, the system will fail. Therefore, it can be expressed as $X_{6} \longrightarrow X_{5} \longrightarrow X_{1}+X_{2}+X_{3}+X_{4}$.

Based on the above system fault paths, a Markov chain is established, as shown in Figure 7.

In this figure, $\lambda_{i}$ represents the probability of occurrence of each bottom event, which can be obtained according to Table 4 . The numbers 1, 2, 3, and 4 represent the state, the arrow represents the state transition, and $F_{a}$ represents the system fault. The state transition path is

$$
\left\{\begin{array}{l}
1 \longrightarrow F a \\
1 \longrightarrow 2 \longrightarrow F a, \\
1 \longrightarrow 3 \longrightarrow F a \\
1 \longrightarrow 3 \longrightarrow 4 \longrightarrow F a .
\end{array}\right.
$$

According to the results of the Markov chain, it can be transformed into the state transition matrix:

$$
T=\left[\begin{array}{ccccc}
-\sum_{i=1}^{6} \lambda_{i} & \lambda_{5} & \lambda_{6} & 0 & \sum_{i=1}^{4} \lambda_{i} \\
0 & -\left(\lambda_{6}+\sum_{i=1}^{4} \lambda_{i}\right) & 0 & 0 & \lambda_{6}+\sum_{i=1}^{4} \lambda_{i} \\
0 & 0 & -\sum_{i=1}^{5} \lambda_{i} & \lambda_{5} & \sum_{i=1}^{4} \lambda_{i} \\
0 & 0 & 0 & -\sum_{i=1}^{4} \lambda_{i} & \sum_{i=1}^{4} \lambda_{i} \\
0 & 0 & 0 & 0 & 0
\end{array}\right] .
$$

Establish Markov differential equation, $t=0 \mathrm{~h}$, $P(0)=\left(\begin{array}{lllll}1 & 0 & 0 & 0 & 0\end{array}\right)^{T}, d P(t) / d t=T^{T} P(t)$, and $P(t)=\left(P_{1}(t)\right.$, $\left.P_{2}(t), P_{3}(t), P_{4}(t), P_{5}(t)\right)^{T}$ : 


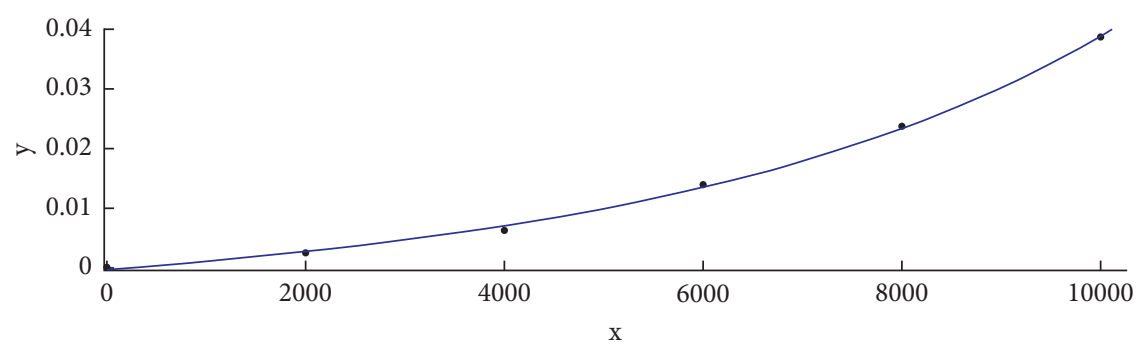

- y vs. $\mathrm{x}$

- Failure rale curve

FIgUre 6: Curve of failure rate over time.

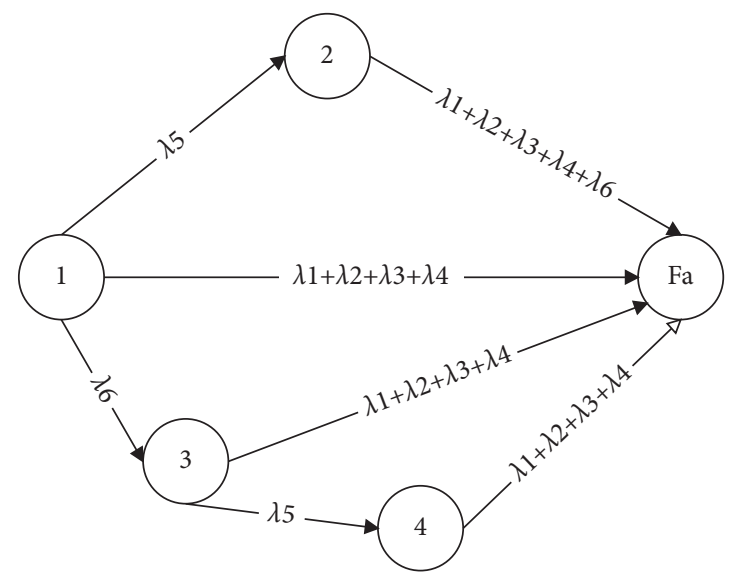

Figure 7: Markov chain.

$$
\begin{aligned}
& P_{1}(t)=\exp \left(-\sum_{i=1}^{6} \lambda_{i} t\right) \\
& P_{2}(t)=\exp \left(-\left(\lambda_{6}+\sum_{i=1}^{4} \lambda_{i}\right) t\right)-\exp \left(-\sum_{i=1}^{6} \lambda_{i} t\right) \\
& P_{3}(t)=\exp \left(-\sum_{i=1}^{5} \lambda_{i} t\right)-\exp \left(-\sum_{i=1}^{6} \lambda_{i} t\right), \\
& P_{4}(t)=\exp \left(-\sum_{i=1}^{4} \lambda_{i} t\right)-\exp \left(-\sum_{i=1}^{5} \lambda_{i} t\right)-\frac{\lambda_{5}}{\lambda_{5}+\lambda_{6}} \exp \left(-\sum_{i=1}^{4} \lambda_{i} t\right)+\frac{\lambda_{6}}{\lambda_{5}+\lambda_{6}} \exp \left(-\sum_{i=1}^{6} \lambda_{i} t\right) \\
& P_{5}(t)=\frac{\lambda_{5}}{\lambda_{5}+\lambda_{6}} \exp \left(-\sum_{i=1}^{6} \lambda_{i} t\right)-\frac{\lambda_{6}}{\lambda_{5}+\lambda_{6}} \exp \left(-\sum_{i=1}^{4} \lambda_{i} t\right)-\exp \left(-\left(\lambda_{6}+\sum_{i=1}^{4} \lambda_{i}\right) t\right)+1 .
\end{aligned}
$$

The failure rate of the system is 0.034375 , which is consistent with the result of the continuous-time T-S dynamic fault tree analysis algorithm.

According to the above results, the continuous-time T-S dynamic fault tree analysis method can directly quantitatively analyze the dynamic fault tree established by the dynamic system and can better describe the static, dynamic, and even multiple states of the system than the general dynamic fault tree analysis method. It expresses the possibility of the occurrence of superior events under the condition that the bottom events occur in different time sequences. In the calculation process, the problem of information explosion that may occur when using the commonly used Markov chain analysis and calculation method 


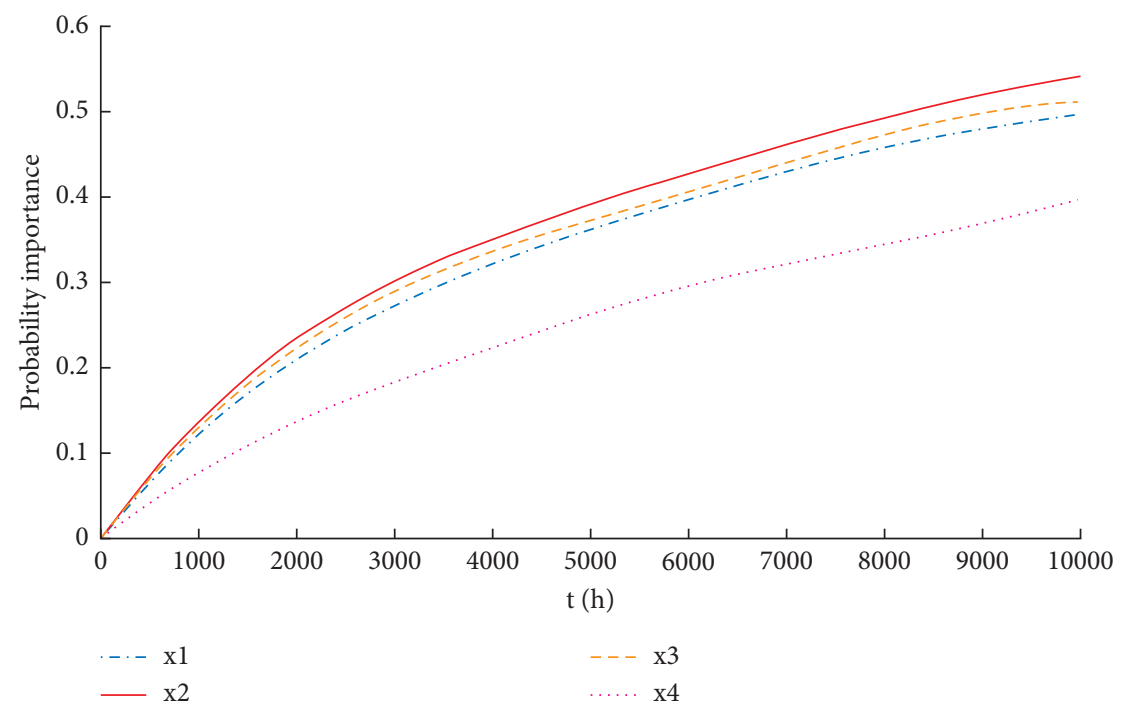

Figure 8: Probability importance change curve.

to solve the highly complex system is avoided. It can be seen that the continuous-time T-S dynamic fault tree analysis method is feasible and has certain advantages.

\subsection{Importance Analysis of Continuous-Time T-S Dynamic} Fault Tree. Importance is used to represent the "contribution" to the top event when the basic event occurs and can quantitatively analyze the impact of the unit on the system reliability. It is used not only for system reliability analysis to find the weak links of the system but also for system reliability improvement, reliability optimization, reliability allocation, and guiding system operation, maintenance, and diagnosis. It is an important index to measure the impact of bottom events on top events. The higher the value, the greater the impact. In the past, the traditional importance analysis of dynamic fault tree cannot realize the change of the influence of bottom events on top events when the importance changes with time. The importance analysis of the continuous-time T-S dynamic fault tree can directly reflect the change of influence degree with time.

3.1.1. Probability Importance. The probability importance formula of continuous-time T-S dynamic fault tree is as follows:

$$
I_{P_{r}\left(X_{i}\right)}=\frac{\partial \int_{0}^{t} f_{Y}(t) \mathrm{d} t}{\partial F_{X_{i}}(t)} .
$$

$f_{Y}(t)$ is the top event failure probability density function, which represents the failure probability of the top event $y$ in the next unit time $t ; F_{X_{i}}(t)$ is the unreliability function of the bottom event $X_{i}$, that is, the cumulative fault distribution function.

Since the probability importance of $X_{5}$ and $X_{6}$ is lower than others, it is not shown in the figure. After calculation, the probability importance of the other four bottom events with time is represented by different line types $X_{1}, X_{2}, X 3$, and $X 4$, respectively, the horizontal axis $t$ represents the time taking $0-10000 \mathrm{~h}$, and the vertical axis represents the probability importance.

Taking the bottom event $X_{2}$ as an example, when $t=10000 \mathrm{~h}$, calculate the probability importance of the event:

$$
\begin{aligned}
I_{P_{r}\left(X_{2}\right)} & =\frac{\partial \int_{0}^{t} f_{Y}(t) \mathrm{d} t}{\partial F_{X_{2}}(t)} \\
& =\left[1-F_{Y_{2}}(t)\right]\left[\left(1-F_{Y_{3}}(t)\right) \times\left(1-F_{X_{1}}(t)\right)\right] .
\end{aligned}
$$

Calculated with data, when $t=10000 \mathrm{~h}, I_{P_{r}\left(X_{2}\right)}=0.54115$. In summary, the results are shown in Figure 8.

As shown in the figure, the probability importance of the overflow valve changes the most with time without oil pipe and oil filter, the Jack is the smallest, and the order from large to small is $X_{2}>X_{3}>X_{1}>X_{4}$. The weak link of the system is the overflow valve. Since the failure rate of each bottom event itself has an impact on the probability importance, the key importance is calculated to ensure the accuracy of the results.

3.1.2. Critical Importance. The critical importance of the continuous-time T-S dynamic fault tree is

$$
I_{C_{r}}^{f}\left(X_{i}\right)=\frac{F_{X_{i}}(t)}{\int_{0}^{t} f_{Y}(t) \mathrm{d} t} I_{P_{r}\left(X_{i}\right)} .
$$

In the formula, the probability distribution function of the bottom event is compared with that of the top event, which effectively reduces the error caused by the large failure rate of the bottom event itself to the importance. The calculated image is shown in Figure 9.

It can be seen from the figure that although the critical importance is lower than the probability importance, it is $X_{1}>X_{3}>X_{2}>X_{4}$ from large to small. According to the analysis results of the two kinds of importance, the failure efficiency of the bottom event $X_{1}$ itself is the largest, which 


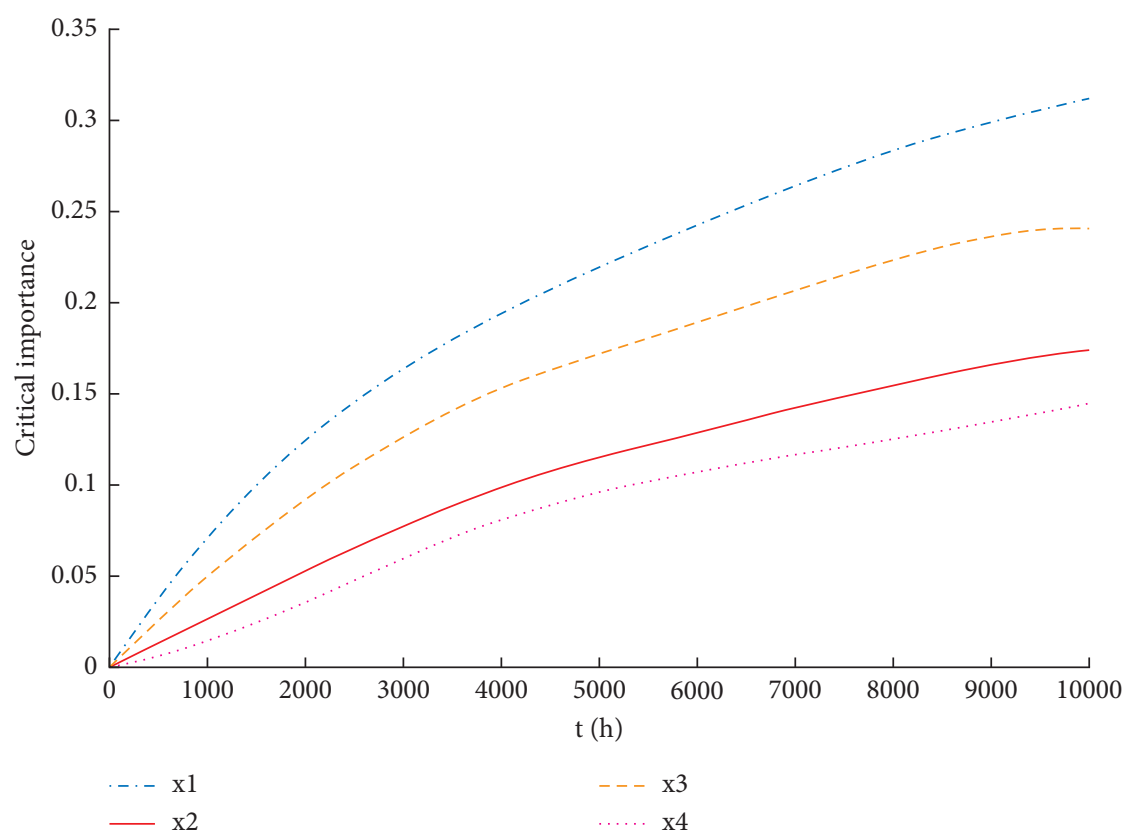

Figure 9: Critical importance.

has an impact on the importance result and changes the impact on the top event. In conclusion, considering the results of critical importance, the weak link of the system is the quantitative hydraulic pump. In fault diagnosis and regular maintenance, it can be tested according to the order of importance.

This section may be divided into subheadings. It should provide a concise and precise description of the experimental results, their interpretation, and the experimental conclusions that can be drawn.

\section{Conclusions}

In this paper, the hydraulic system of the tape winding trolley, for example, the application of the continuous-time T-S dynamic fault tree analysis algorithm to the dynamic system reliability quantitative analysis, and the results compared with Markov chain analysis results verify the feasibility of the method and illustrate that the continuoustime T-S has the advantage of dynamic fault tree analysis method. The importance of the system is analyzed, the influence degree of different components on the hydraulic system fault with time is shown, and the weak links of the system are pointed out to provide methods for fault maintenance and prevention.

\section{Data Availability}

The component failure data used to support the findings of this study are included within the paper.

\section{Conflicts of Interest}

The authors declare that there are no conflicts of interest regarding the publication of this paper.

\section{Acknowledgments}

This research was funded by the National Natural Science Foundation of China (nos. 51965051 and 71761030) and the Natural Science Foundation of Inner Mongolia Autonomous Region, China (no. 2020MS05065).

\section{References}

[1] Q. Ma, J. Ge, M. R. M. Rejab et al., "Fabrication of the carbon fiber reinforced plastic (CFRP) cone tube through the laboratory-scale 3-axis winding machine," Materials Today Proceedings, vol. 46, 2020.

[2] Bo Deng, Y. Shi, Yu Tao, and Z. Pan, "Influence mechanism and optimization analysis of technological parameters for the composite prepreg tape winding process," Polymers, vol. 12, no. 8,2020

[3] H. Song, H.-Y. Zhang, and C. W. Chan, "Fuzzy fault tree analysis based on T-S model with application to INS/GPS navigation system," Soft Computing, vol. 13, no. 1, 2009.

[4] C. Y. Yao, C. L. Wang, D. N. Chen, X. Wei, and S. J. Lu, "Continuous time T-S dynamic fault tree analysis method," Journal of Mechanical Engineering, vol. 56, no. 10, pp. 244-256, 2020.

[5] A. S. Cheliyan and S. K. Bhattacharyya, “"Dynamic fault tree analysis of dynamic positioning system using Monte Carlo approach"," SAfety in Extreme Environments: People, Risk and Security, vol. 1, no. 3, 2019.

[6] Engineering - Safety Engineering, "New safety engineering findings from university of hull described "A hierarchical approach for dynamic fault trees solution through semimarkov process," Journal of Technology \& Science, vol. 69, no. 3, pp. 986-1003, 2020.

[7] O. Yevkin, "An efficient approximate Markov chain method in dynamic fault tree analysis," Quality and Reliability Engineering International, vol. 32, no. 4, 2016.

[8] S. Gupta and K. Singh, "Analysis of a Markov chain system model of a power generating system composed of generating 
units and transformer units," Life Cycle Reliability and Safety Engineering, no. 10, pp. 273-283, 2021.

[9] S. Morteza, C. Felician, and N. Daniel, "Integration of Hidden Markov Modelling and Bayesian Network for fault detection and prediction of complex engineered systems," Reliability Engineering \& System Safety, vol. 215, 2021.

[10] J. Mi, Y.-F. Li, W. Peng, and H.-Z. Huang, "Reliability analysis of complex multi-state system with common cause failure based on evidential networks," Reliability Engineering \& System Safety, vol. 174, 2018.

[11] L. Zong-Yuan, G.-J. Jiang, C. Hong-Xia, H.-B. Li, H.-H. Sun, and Y. Mohammad, "Reliability analysis of special vehicle critical system using discrete-time bayesian network," Mathematical Problems in Engineering, vol. 2021, Article ID 5579218, 9 pages, 2021.

[12] Y. Guo, M. Zhong, C. Gao, H. Wang, X. Liang, and H. Yi, “A discrete-time Bayesian network approach for reliability analysis of dynamic systems with common cause failures," Reliability Engineering \& System Safety, vol. 216, 2021.

[13] G. Yan, "Modeling and reliability analysis of fuzzy random model of end-bearing piles," International Journal of Frontiers in Engineering Technology, vol. 0, no. 2, p. 3, 2021.

[14] Li Xin, X. Mu, and Z. Yang, "Reliable dissipative control for fuzzy singular semi-markovian jump systems with modedependent delays and randomly occurring uncertainties," Journal of the Franklin Institute, vol. 358, no. 5, 2021.

[15] M. Jirgl, M. Havlikova, Z. Bradac, and L. Stastny, "Monte Carlo reliability analysis of systems with a human operator," IFAC-PapersOnLine, vol. 49, no. 25, 2016.

[16] Yu Liu, J. Li, T. Jiang, Z. Zhang, Z. Shi, and Bo Yang, “A rapid Monte Carlo reliability evaluation method for integrated energy systems based on transformer," Journal of Physics: Conference Series, vol. 2087, no. 1, 2021.

[17] Y. Wu and J. Tao, "Dynamic fault tree analysis of loader hydraulic system based on T-S model," Hydraulics and Pneumatics, no. 11, pp. 171-176, 2020.

[18] F. Gong, Z.-kai Yuan, and Z.-xin He, "A fuzzy reliability analysis and simulation method of hydraulic system," in Proceedings of the 2017 5th International Conference on Mechatronics, Materials, Chemistry and Computer Engineering, July 2017.

[19] W. Chen, H. Wang, G. H. Zhang, C. T. Wang, and G. Q. Zhong, “"Evaluation of tunnel collapse vulnerability based on T-S fuzzy fault tree and bayesian network," Journal of Shanghai Jiao Tong University, vol. 54, no. 8, pp. 820-830, 2020.

[20] L. Zhang, R. J. Zhang, and S. X. Xin, "Importance analysis method of fuzzy fault tree based on T-S modle and application in hydraulic system," Applied Mechanics and Materials, vol. 470, 2013. 